\title{
Self-powered Wireless Occupancy Detector based on Flexible Piezoelectric Film
}

\author{
Sherif Keddis, Rafik Mitry, Norbert Schwesinger \\ Technical University of Munich, Professorship for Microstructured Mechatronic Systems \\ Arcisstr. 21, 80333 Munich, BY, Germany \\ sherif.keddis@tum.de; rafik.mitry@tum.de; schwesinger@tum.de
}

\begin{abstract}
Harvesting mechanical energy from footsteps or other impacts is a possibility to enable wireless autonomous sensor networks. These can be used for a highly efficient control of connected devices such as lights, security systems, air conditioning systems or other smart home applications. They can also be used for accurate location or occupancy monitoring. Converting the mechanical energy into useful electrical energy can be achieved using the piezoelectric effect offering simple harvesting setups and low deflections. The challenge facing piezoelectric transducers is the achievable amount of energy per impact in the lower $\mathrm{mJ}$ range and the management of such low energies. Simple setups for energy extraction such as a full wave bridge directly connected to a capacitor are problematic due to the mismatch between high impedance sources and low impedance storage elements. Efficient energy circuits for piezoelectric harvesters are commonly designed for vibration harvesters and require periodic inputs with predictable frequencies. Due to the sporadic nature of impact harvesters, such circuits are not well suited, since they reduce the available output. This paper discusses a piezoelectric energy harvester using a polyvinylidenfluoride (PVDF) film to capture human step-energy. Focus of this paper is a suitable energy management circuit. Furthermore, a simple wireless sensor node functioning as a switch is implemented and tested in order to validate the energy harvester capabilities to power practical applications. The implemented wireless switch consumes $58 \mu \mathrm{J}$ to transmit. The energy harvester stores about $122 \mu \mathrm{J}$ per step on a $220 \mu \mathrm{F}$ capacitor, which is sufficient to cover the required energy and recharge a secondary battery over time. This self-sufficient approach enables a maintenance-free and indefinite operation of such wireless sensor nodes opening a wide field of new applications.
\end{abstract}

Keywords: Autonomous sensors, Building automation, Energy harvesting, IoT, Piezoelectricity, PVDF.

\section{Introduction}

Artificial lightning makes up for $19 \%$ of the global energy consumption [1]. This number increases to $40 \%$ when related to the total consumption of buildings [2]. Another $50 \%$ is consumed by heating, ventilation and air conditioning (HVAC) systems [3]. These high consumption rates can be reduced by up to $30 \%$ through the use of efficient occupancy sensing and control [4]. This is mostly done using passive infrared (PIR) sensors that detect occupancy due to changes in thermal images [5]. When a change is detected, indicating occupancy, the lights can be switched on for a specified period. When no motion is detected within this period, the system regards the space as unoccupied and the lights are switch back off. Still or barely moving occupiers of a space can cause false interpretation of such systems. To avoid that, the "On" period is set long enough to increase the probability of detecting motion in an occupied space. This leads to an energy waste every time a space is cleared [6]. Supplementing the PIR sensors with other detection mechanisms and algorithms can improve the reliability of the occupancy detection but also increases the complexity and consumption [7], [8]. Using pressure sensors, occupancy can also be detected to efficiently control the building [9]. Piezoelectricity, as a common effect to measure changes in pressure and therefore, detect occupancy, offers an additional advantage. The mechanical strain of such materials, due to changes in pressure, generates electrical charges that can harvested for autonomous operation of the monitoring system. This eliminates the complex wiring associated with grid-powered high resolution monitoring systems or the maintenance requirements for replacement of battery-powered sensors. The feasibility of the energy harvesting capability of piezoelectric materials for powering occupancy detection systems or similar applications autonomously is discussed in this paper. 


\section{Related Work}

The feasibility of an energy harvester to supply certain systems is strongly dependent on the available input energy, the electrical output energy and the required energy by the application. Typical sources for energy harvesting in buildings and their respective potential power output are artificial lighting, thermal gradients, mechanical energies in form of vibrations, airflow as well as electromagnetic waves. The reported output of the corresponding harvesters reaches $1 \mathrm{~mW}$, $10 \mathrm{~mW}, 70 \mu \mathrm{W}, 300 \mathrm{~mW}$ and $1 \mu \mathrm{W}$, respectively [10]. Apart from being an energy source, some of these inputs can be used to detect occupancy as well. The mechanical impact energy of human footsteps is one example that has received much research attention. This can be done using mobile approaches attached to the human [11]-[15], or fixed approaches implemented in the ground [16]-[23]. Mobile approaches require each occupier to be equipped with a sensor/harvester. This is impractical since the number of occupiers can be very large and is often unpredictable. This approach also allows for more human error if the sensors are not worn properly or forgotten. Therefore, the fixed approach is more suitable for occupancy detection for purposes of building automation, as it is tied to specific locations, reducing the number of required sensors. In addition, the detection algorithm would be very simple and conclusive.

Fixed floor harvesters can be implemented using several conversion principles. Using the electromagnetic effect, Duarte et al. managed to generate up to $0.6 \mathrm{~W}$ at frequency of one step per second [24]. Adding a flywheel to the generator to increase the harvesting efficiency allowed Liu et al. to reach $1.8 \mathrm{~W}$ at the same step frequency [18]. Commercial implementations by Energy Floors ${ }^{\circledR}$ and Pavegen ${ }^{\circledR}$ claim even higher outputs of $4 \mathrm{~W}$ and $5 \mathrm{~W}$, respectively [21], [25]. These rather high outputs come at the cost of large strokes required for the relative movement between the magnet and the coil. Large deflections can affect the human movement and be considered uncomfortable. This lead Sharpes et al. to implement a harvester using the piezoelectric effect [20]. Using lead zirconate titanate (PZT) cymbals, they were able to harvest $1 \mathrm{~mJ}$ from a step load of $410 \mathrm{~N}$ with a deflection of $0.5 \mathrm{~mm}$. The same amount was harvested by Nakajima et al. using multilayered polyvinylidenfluoride (PVDF) and an amplified load of $4.2 \mathrm{kN}$ [19]. The dimensions required to amplify the forces using a lever were $50 \times 50 \mathrm{~cm}^{2}$. Another approach to transfer the vertical step forces into multilayered PVDF films was implemented by Bischur using hinges [26]. At a load of $400 \mathrm{~N}$ he was able to harvest $90 \mu \mathrm{J}$ per step. The low output was a result of fabrication issues that lead to mechanical failure of the device at higher strains. This design was tweaked as a part of the ongoing research project. The improved harvester and its output are discussed in the following section.

The output of energy harvesters should cover the consumption of the desired application in order to substantially prolong its operational lifetime compared to other supply forms, such as batteries. Ultra-low-power wireless PIR sensors consume about $4.7 \mathrm{~mW}$ when active. With an average of 10 detected movements per hour, the lifetime of such sensor is calculated to eight years when powered with a 3 V lithium-ion coin cell (CR2032) [27]. This calculates to an average power consumption of about $9 \mu \mathrm{W}$ for the entire operational lifetime which can easily be supplied by harvesters mentioned earlier if the input energy is constantly available. Hybrid detection systems using PIR and cameras have a higher consumption since the camera alone consumes between 50 and $250 \mathrm{~mW}$ [28]. This consumption rate is too high for most energy harvesters. Using floor harvesters to detect occupancy eliminates the sensor consumption, reducing the total consumption to the wireless transmission of the detected events. Ultra-low-power microcontrollers (MCU) and transmitters consume in active-mode as low as $600 \mu \mathrm{W}$ and $20 \mathrm{~mW}$, respectively [29]. Because the active duration is as low as a couple of milliseconds, the average consumption is reduced to the range of $60 \mu \mathrm{J}$ per detection. For a scenario with the same 10 movements per hour, this would lead to an average consumption of $170 \mathrm{nW}$. The limiting factor in this case is the consumption during sleep mode of about $1.5 \mu \mathrm{W}$. This amount should still be easily covered by floor harvesters.

\section{Multilayered PVDF Floor Harvester}

The initial design reported by Bischur uses two PVDF layers with two aluminium foils as electrodes. This multilayer is wound several times to increase the active volume and welded to avoid unfolding. To utilize the piezoelectric transverse effect, the step forces are transferred into the active layers using a hinge placed inside the PVDF wrap. Fig. 1 shows this concept on the left as well as the hinge design on the right. 

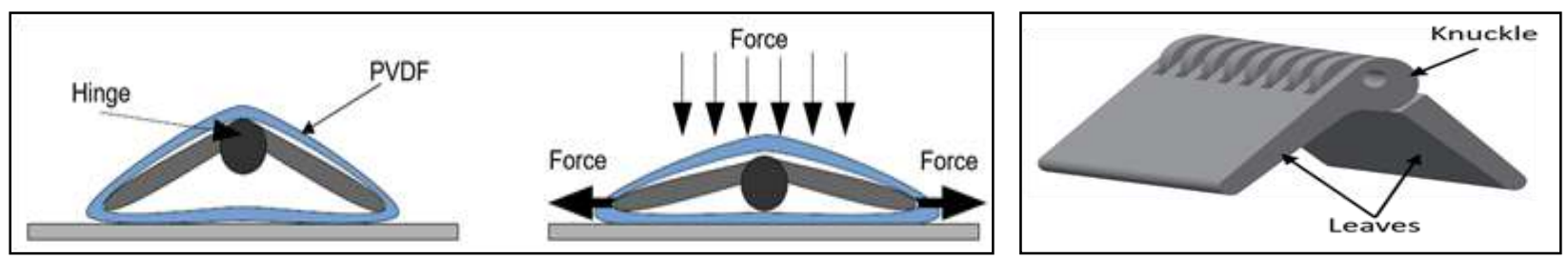

Fig. 1: Hinge to transfer the forces and enable the piezoelectric transversal effect [26] (left) and the improved hinge design (right).

As part of the improvements on Bischur's work, the hinge is fabricated by injection moulding of polyoxymethylene (POM) to ensure a smooth gliding of the film over the edges compared to hinges of other materials. Further improvements include an automated fabrication of the PVDF wrap using film capacitor technologies by the German manufacturer FTCAP ${ }^{\circledR}$. The improved design as well as the dimensions of the proposed harvester are reported in [30]. When the harvester is loaded by a vertical mechanical force, the hinge is depressed. The increased circumference stretches the PVDF film resulting in a polarization change. The stroke of the hinge and the resulting elongation of the active layers depends on the applied force and the windings of the PVDF wrap. The maximum elongation of $1.3 \%$ is achieved, when the hinge is fully depressed and the knuckle reaches the ground. The energy output based on the mechanical input force for wraps with different number of windings is presented in Fig. 2. Typical dynamic forces during human locomotion around $640 \mathrm{~N}$ [31] result in an energy output of $3.7 \mathrm{~mJ}$ per step (loading and release) using a wrap with three windings measured across a resistive load of $5 \mathrm{M} \Omega$ [30].

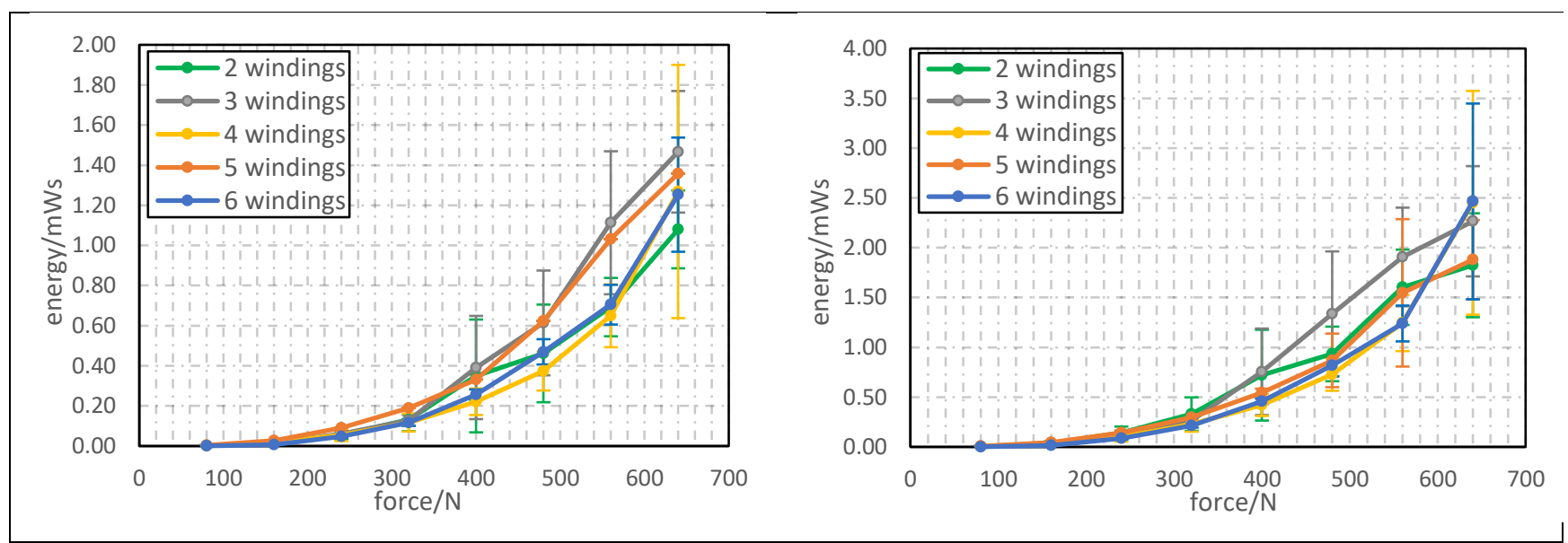

Fig. 2: Energy output for different winding numbers based on the input force for loading (left) and release (right) [30].

\section{Wireless Occupancy Detector}

To prove the feasibility of the proposed harvester for powering practical applications, a simple wireless occupancy detector was implemented. When an event occurs, stepping on or off the harvester, the energy is extracted through a management circuit and stored to supply the remaining components. Since the piezoelectric effect delivers an alternating signal, the output voltage of the harvester is used to detect the type of event (i.e., step on or off). When an event is detected, the MCU is triggered to wake up and transmit the detected event, then returns to sleep. The basic structure is illustrated in Fig. 3. 


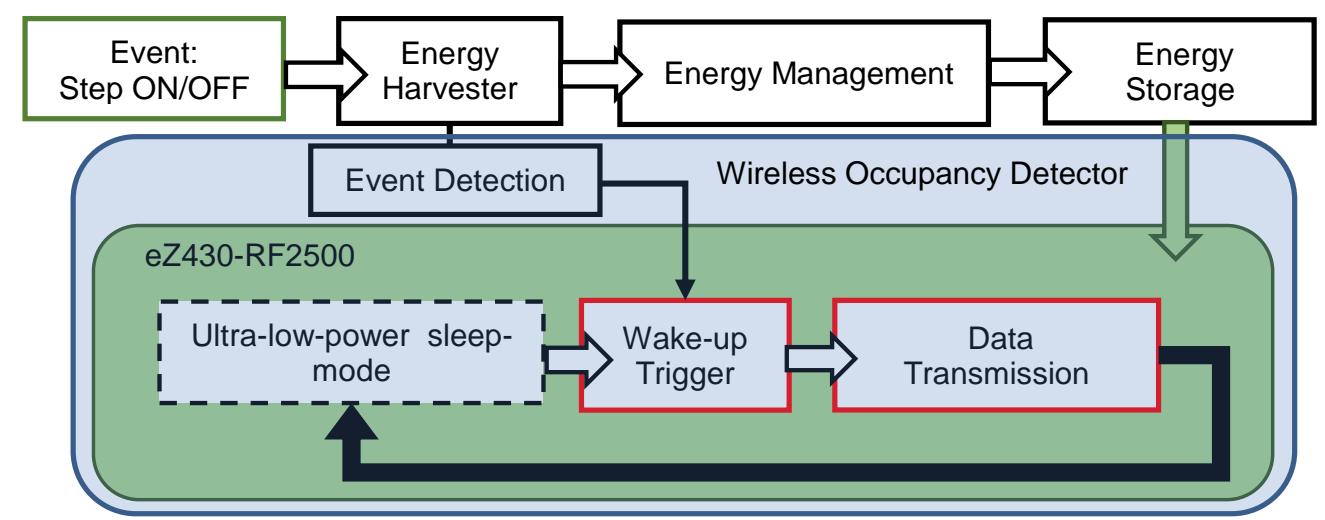

Fig. 3: Basic structure of the harvester-powered wireless occupancy detector.

\subsection{Implementation}

The event detection is implemented using two voltage dividers and two n-Channel MOSFETs as seen in Fig. 4. When the harvester is stepped on, the voltage rises across R3 and R2. The divider acts as a threshold to prevent false detection if the output signal is not high enough. When the voltage across R2 rises above the MOSFET's gate threshold, M1 is switched on and conducts pulling the first GPIO pin of the MCU from its high state (due to the pull-up resistor $\mathrm{R} 1)$ to ground. This falling edge across the pin is used to trigger an interrupt and to wake the MCU from sleep to transmit the detection of a step-On. The same functionality is observed when the harvester is released (i.e., step-Off) except the voltage rises across R6 and R5 to switch M2 on and pull the second GPIO pin to ground. This triggers the MCU to wake up and run the step-Off routine. The voltage levels across each divider and their corresponding GPIO pins is shown in Fig. 5. The eZ430-RF2500 development board by Texas Instruments ${ }^{\circledR}$ is used as an off-the-shelf solution for the MCU and RF-module and was chosen based on ultra-low-power characteristics and availability [29].

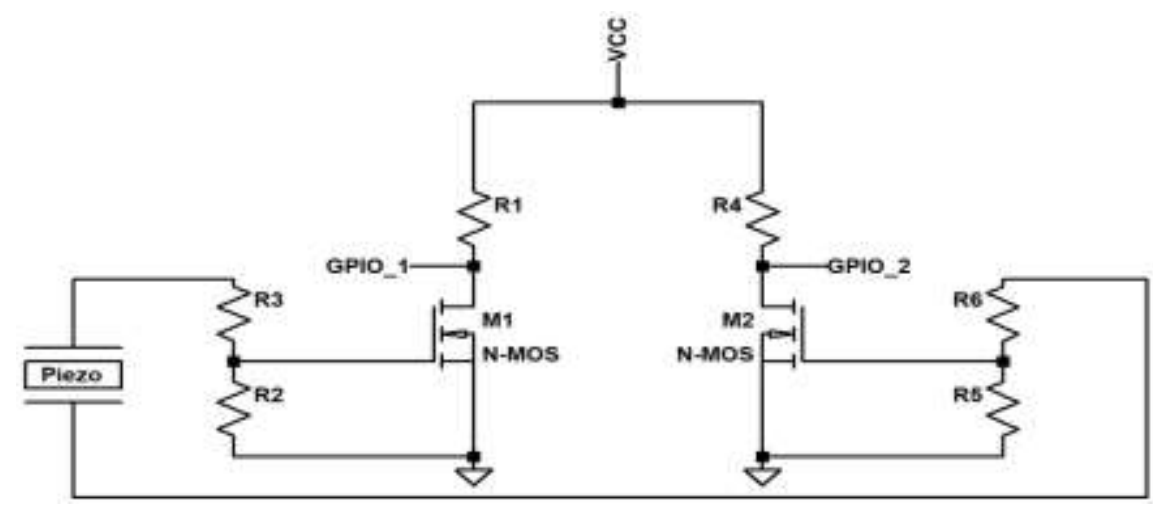

Fig. 4: Circuit to detect the event (i.e., step on or off). 


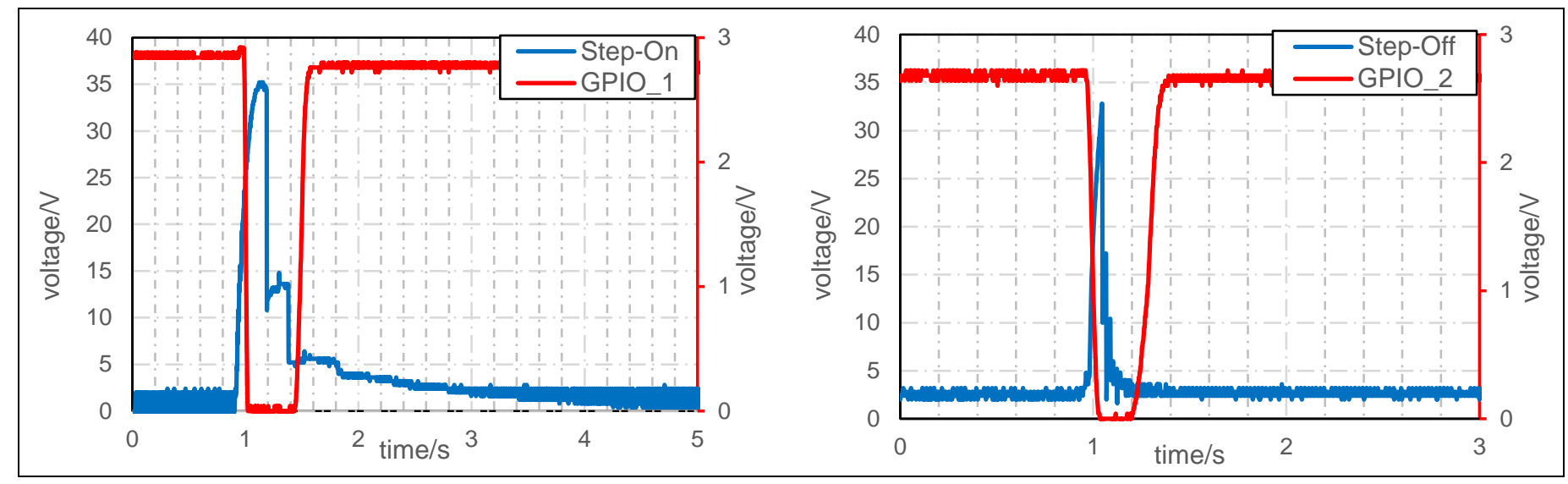

Fig. 5: Harvester output and corresponding GPIO states during step-On (left) and step-Off (right).

\subsection{Consumption}

The total energy consumption of the implemented application is divided into two parts. The circuit consumption for the event detection and the consumption of the eZ430-RF2500 target board. The circuit consumption is due to the current flowing through the voltage dividers, the MOSFET's consumption as well as the current flowing through the pull-up resistor when the MOSFET is conducting. To measure this consumption, the stored energy delivered by the harvester on a capacitor was measured once without the detection circuit shown in Fig. 4 and another time with it. The difference was considered to be the circuit consumption $E_{c}$. However, the pull-up resistors were not connected to the supply voltage during the second measurement so their consumption was not regarded. In order to include their consumption, the supply voltage $V_{c c}$ was used in combination with the resistance $R$ and on-period of the MOSFET $t_{o n}$ to calculate the energy $E_{p}$ following:

$$
E_{p}=\frac{V_{c c}^{2}}{R} * t_{o n}
$$

As for measuring the consumption of the target board, a shunt resistance $R_{S}$ of $8 \Omega$ was used in series with the input and the effective voltage drop $V_{r m s}$ across it was measured to determine the average current consumption. Fig. 6 shows the voltage course across the shunt resistance. To reduce the energy consumption of the target board, the transmission power was reduced from $0 \mathrm{dBm}$ (blue) to $-10 \mathrm{dBm}$ (red).

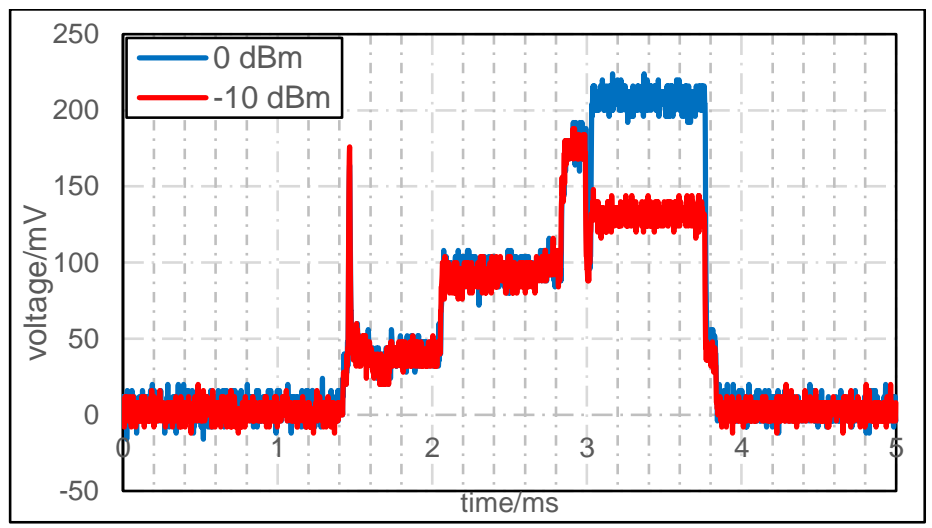

Fig. 6: Current consumption of the target board measured as a voltage over an $8 \Omega$ shunt resistance during an event transmission at $0 \mathrm{dBm}$ and $-10 \mathrm{dBm}$. 
This current along with the supply voltage and the transmission time $t_{T x}$ are used to calculate the board consumption $E_{b}$ following Eq. (2). Eq. (3) shows the total energy consumption of the application $E_{\text {tot }}$ as the sum of all partial and averages $80 \mu \mathrm{J}$ for a transmission with $-10 \mathrm{dBm}$ and a supply voltage of $2.1 \mathrm{~V}$ based on three test runs.

$$
\begin{gathered}
E_{b}=\frac{V_{r m s}}{R_{s}} * V_{c c} t_{T x} \\
E_{t o t}=E_{c}+E_{p}+E_{b}
\end{gathered}
$$

\section{Proof of Concept}

In order to be able to run the proposed application in section 4 using the discussed harvester from section 3 , the output energy has to be managed efficiently (i.e., rectified, buffered and regulated). The rectification is achieved using a standard off-the-shelf full-wave bridge rectifier, since the voltage output reaches several tens of Volts. This makes the voltage drop negligible and advantages of alternative rectification methods obsolete. Directly connecting a capacitor, as a typical extraction circuit, is inefficient because piezoelectric harvesters, especially PVDF-based ones, are high impedance sources resulting in a large impedance mismatch. Therefore, a simple self-powered peak detector circuit was used between the harvester and the storage element [32]. The idea is to disconnect the load (i.e., the storage capacitor) from the harvester allowing it to fully charge. When the voltage reaches its peak, the switch is closed and the charge flows into the storage. The capacitor is combined with an inductor and a diode to form an oscillating circuit. It acts as an electrical flywheel and enables a more efficient extraction of the charge. The complete circuit is drawn in Fig. 7 along with the voltage courses for the rectified output and across the storage capacitor.

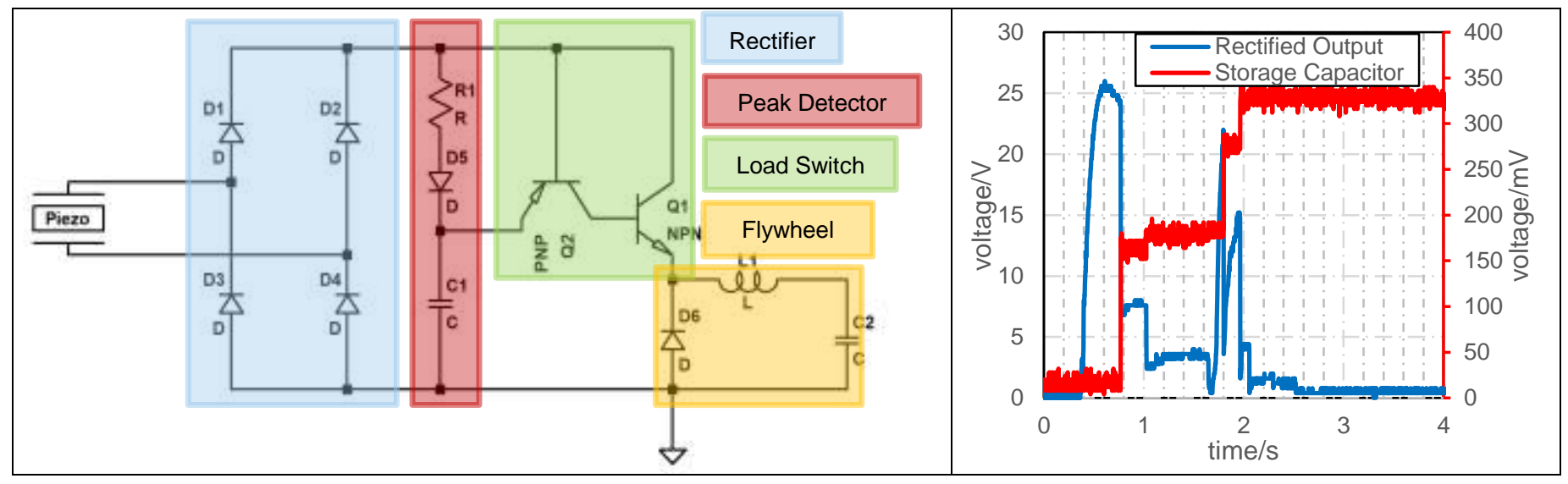

Fig. 7: Efficient charge extraction circuit for impact harvesters (left) and courses of the rectified voltage and the capacitor voltage (right).

Choosing the capacitor size proposed a challenge since large capacitors would only charge to several hundreds of millivolts, which is too low to operate the load. Small capacitors, on the other hand, reach high enough voltages but store limited energy. This results in a too quick voltage breakdown as soon as current is drawn. As a compromise, a large $220 \mu \mathrm{F}$ capacitor was chosen and pre-charged to the correct operating voltage using an external source. Before disconnecting the supply voltage, the MCU is initialized and enters sleep mode. In practice, the pre-charged large capacitor can be replaced by a battery, especially to benefit from the high energy density and the low leakage current.

In order to determine the capabilities of the energy harvester to power the proposed application, the voltage across the capacitor was observed while the harvester was loaded periodically with a force of $320 \mathrm{~N}$. As a response, the harvester charges the storage capacitor and triggers the application. Fig. 8 shows the voltage course across the storage capacitor. Due to the re-charging of the capacitor through the harvester, the transmission number was increased to 216 
(red curve) compared to only 10 if the application was running off the pre-charged storage and no harvester connected (blue curve). The zoomed-in graph on Fig. 8 (right) shows the consumption (red) and charging (blue) during loading and releasing. Although less energy is re-charged during loading, the average energy per event (i.e., loading or release) is $61 \mu \mathrm{J}$ at $2.3 \mathrm{~V}$. The average consumed energy for that operating voltage is $58 \mu \mathrm{J}$ which is less than the result of equation (3) since the consumption of the circuit $E_{c}$ is deducted from the charging energy that reaches the capacitor. At higher operating voltages, the consumption increases, explaining the drop in capacitor voltages. The voltage drops eventually below the operating range due to tolerances in harvester performance and a too small overhead of $3 \mu \mathrm{J}$. For applications where a transmission per complete step is sufficient, the available energy of $122 \mu \mathrm{J}$ should be enough to cover the consumption and re-charge the storage for an indefinite time.

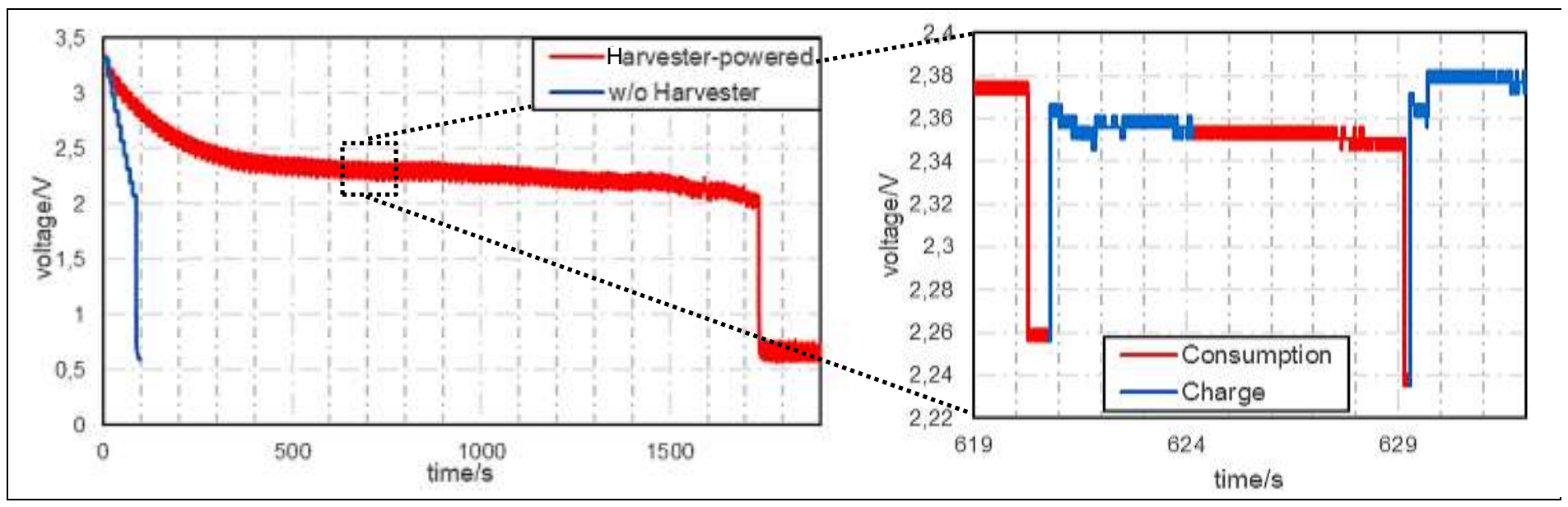

Fig. 8: Voltage course across the $220 \mu \mathrm{F}$ storage capacitor during application.

\section{Conclusion}

The implemented wireless switch proved the feasibility of the proposed piezoelectric impact harvester for certain applications with a consumption in the range of $100 \mu \mathrm{J}$. For proper operation, the storage element needs to be large and prebiased (e.g., rechargeable battery). The harvester can re-charge the consumed energy and counter the self-discharge of the battery, prolonging its lifetime indefinitely.

Future work includes characterization of the aging and long-term performance of proposed harvester.

\section{References}

[1] A. Bahga and V. Madisetti, Internet of Things: A hands-on approach. Vpt, 2014.

[2] Energy Information Administration, "Commercial buildings energy consumption survey," 2003.

[3] L. Pérez-Lombard, J. Ortiz, and C. Pout, "A review on buildings energy consumption information," Energy Build., vol. 40, no. 3, pp. 394-398, 2008.

[4] V. Garg and N. K. Bansal, "Smart occupancy sensors to reduce energy consumption," Energy Build., vol. 32, no. 1, pp. 81-87, Jun. 2000.

[5] C. de Bakker, M. Aries, H. Kort, and A. Rosemann, "Occupancy-based lighting control in open-plan office spaces: A state-of-the-art review," Build. Environ., vol. 112, pp. 308-321, 2017.

[6] X. Wang, T. Tjalkens, and J. P. Linnartz, "Smart office lighting control using occupancy sensors," in Proc. 2017 IEEE 14th Int. Conf. Networking, Sens. Control. ICNSC 2017, pp. 453-458, 2017.

[7] B. Dong, K. P. Lam, R. Zhang, D. Benitez, B. Andrews, M. Hoeynck, Y. S. Chiou, "An information technology enabled sustainability test-bed (ITEST) for occupancy detection through an environmental sensing network," Energy Build., vol. 42, no. 7, pp. 1038-1046, 2010.

[8] J. Hutchins, A. Ihler, and P. Smyth, "Modeling count data from multiple sensors: a building occupancy model," in 
Computational Advances in Multi-Sensor Adaptive Processing, 2007. CAMPSAP 2007. 2nd IEEE International Workshop on, 2007, pp. 241-244.

[9] X. Guo, D. K. Tiller, G. P. Henze, and C. E. Waters, "The performance of occupancy-based lighting control systems: A review," Light. Res. Technol., vol. 42, no. 4, pp. 415-431, 2010.

[10] J. W. Matiko, N. J. Grabham, S. P. Beeby, and M. J. Tudor, "Review of the application of energy harvesting in buildings," Meas. Sci. Technol., vol. 25, no. 1, 2014.

[11] J. Granstrom, J. Feenstra, H. A. Sodano, and K. Farinholt, "Energy harvesting from a backpack instrumented with piezoelectric shoulder straps," Smart Mater. Struct., vol. 16, no. 5, pp. 1810-1820, 2007.

[12] N. S. Shenck and J. A. Paradiso, "Energy scavenging with shoe-mounted piezoelectrics," IEEE Micro, vol. 21, no. 3, pp. 30-42, 2001.

[13] J. Kymissis, C. Kendall, and J. Paradiso, "Power harvesting in shoes," in Proc. 2nd IEEE Int. Symp. Wearable Comput., pp. 132-140, 1998.

[14] H. Kalantarian and M. Sarrafzadeh, "Pedometers without batteries: An energy harvesting shoe," IEEE Sens. J., vol. 16, no. 23, pp. 8314-8321, 2016.

[15] J. Zhao and Z. You, "A Shoe-Embedded Piezoelectric Energy Harvester for Wearable Sensors," Sensors, vol. 14, no. 7, pp. 12497-12510, 2014.

[16] E. Bischur and N. Schwesinger, "Energy harvesting in floors," Jpn. J. Appl. Phys., vol. 1, pp. 45-55, 2010.

[17] O. Puscasu, N. Counsell, M. R. Herfatmanesh, R. Peace, J. Patsavellas, and R. Day, "Powering Lights with Piezoelectric Energy-Harvesting Floors," Energy Technol., vol. 6, no. 5, pp. 906-916, 2018.

[18] M. Liu, R. Lin, S. Zhou, Y. Yu, A. Ishida, M. McGrath, B. Kennedy, M. R. Hajj, L. Zuo, "Design, simulation and experiment of a novel high efficiency energy harvesting paver," Appl. Energy, vol. 212, no. February, pp. 966-975, 2018.

[19] T. Nakajima, K. Okaya, K. Ohta, T. Furukawa, and S. Okamura, "Performance of piezoelectric power generation of multilayered poly(vinylidene fluoride) under high mechanical strain," Jpn. J. Appl. Phys., vol. 50, no. 9, pt. 3, 2011.

[20] N. Sharpes, D. Vučković, and S. Priya, "Floor Tile Energy Harvester for Self-Powered Wireless Occupancy Sensing," Energy Harvest. Syst., vol. 3, no. 1, pp. 43-60, 2016.

[21] M. Smit, "Human-powered small-scale generation system for a sustainable dance club," no. July, pp. $20-26,2011$.

[22] J. C. Brezet et al., "Floor suitable for generating, converting and/or storing energy." Google Patents, 09-Oct-2012.

[23] C. He, W. Zhu, B. Chen, L. Xu, T. Jiang, C. B. Han, G. Q. Gu, D. Li, Z. L. Wang, "Smart Floor with Integrated Triboelectric Nanogenerator As Energy Harvester and Motion Sensor," ACS Appl. Mater. Interfaces, vol. 9, no. 31, pp. 26126-26133, 2017.

[24] F. Duarte, A. Ferreira, and J. Champalimaud, "Waynergy Vehicles-An Innovative Pavement Energy Harvest System," in 2nd International Congress on Energy Efficiency and Energy Related Materials (ENEFM2014), 2015, pp. 343-348.

[25] "Pavegen." [Online]. Available: www.pavegen.com/

[26] E. Bischur, "Energy Harvesting auf Basis monoaxial gereckter PVDF Folie und der Technologie von Folienkondensatoren," 2016.

[27] Texas Instruments, "Ultra-Low-Power Wireless PIR Motion Detector for Cost- Optimized Systems Reference Design," Data Sheet, no. February, pp. 1-36, 2017.

[28] K. W. Agyeman, "Low-power motion detection and building control using Computer Vision."

[29] Texas Instruments, "eZ430-RF2500 Development Tool User ' s Guide," 2015. [Online]. Available: www.ti.com/lit/ug/slau227f/slau227f.pdf.

[30] S. Keddis and N. Schwesinger, "Piezoelectric pressure harvester for autonomous sensors," 2019.

[31] R. Cross, "Standing, walking, running, and jumping on a force plate," Am. J. Phys., vol. 67, no. 4, pp. 304-309, 1999.

[32] J. Liang, W. Liao, and S. Member, "Improved Design and Analysis of Self-Powered Synchronized Switch Interface Circuit for Piezoelectric Energy Harvesting Systems," IEEE Trans. Ind. Electron., vol. 59, no. 4, pp. 1950-1960, 2012. 